MIDPI

sciforum
MOL2NET-07, Conference on Molecular, Biomedical, and Computational Sciences and Engineering, ISSN: 2624-5078, MDPI SciForum, Basel, Switzerland, 2021, 7th ed.

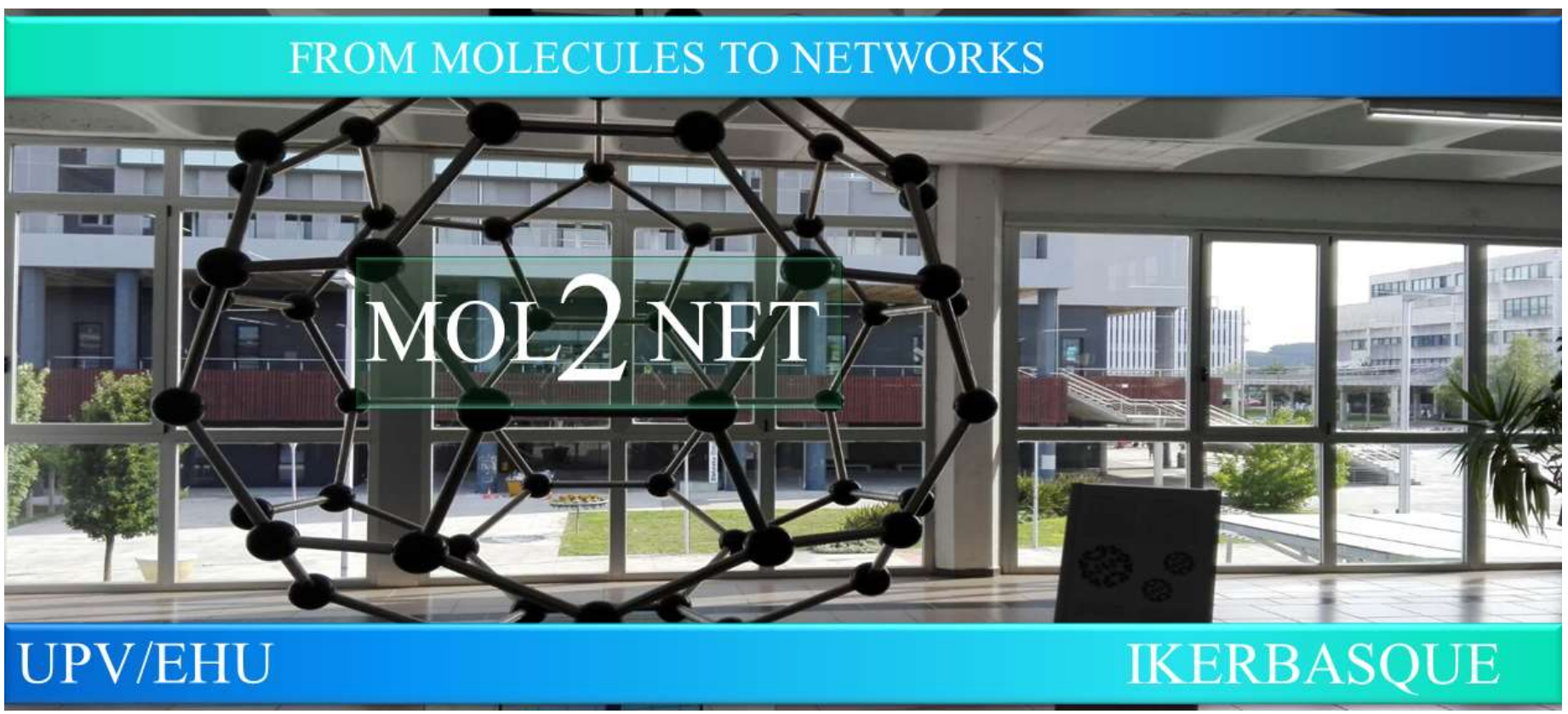

\title{
MOL2NET-07, Conference on Molecular, Biomedical, and Computational Sciences and Engineering, ISSN: 2624-5078, MDPI SciForum, Basel, Switzerland, 2021, 7th ed.
}

Marianne Garber ${ }^{\mathrm{a}, \mathrm{b}}$, Alexander Tropsha ${ }^{\mathrm{c}}$, Francoise Dumas ${ }^{\mathrm{d}}$, Jerzy Leszczynski ${ }^{\mathrm{e}}$, Maria Natália D. S. Cordeiro ${ }^{\mathrm{f}}$, Jia Zhou ${ }^{\mathrm{g}}$, Bakhtiyor Rasulev ${ }^{\mathrm{h}}$, Ivan Ubarretxena ${ }^{\mathrm{i}, \mathrm{r}}$, Peter Langer ${ }^{\mathrm{j}}$, Yasset Perez-Riverol ${ }^{\mathrm{k}}$, David Quesada ${ }^{1}$, James R. Green ${ }^{\mathrm{m}}$, Sonia Arrasate ${ }^{\mathrm{n}}$, Gerardo J Ruiz-Mercado ${ }^{\circ}$, Eugene Muratov ${ }^{\mathrm{c}}$, Dario Greco $^{\mathrm{p}}$, Nuria Sotomayor ${ }^{\mathrm{n}}$, Marcus Tullius Scotti ${ }^{\mathrm{q}}$, Esther Lete ${ }^{\mathrm{n}}$, Humberto Gonzalez-Diaz ${ }^{\mathrm{i}, \mathrm{n}, \mathrm{r},{ }^{*}}$

${ }^{a}$ Faculté des Sciences et Technologies, Université de La Rochelle, La Roche, France. ${ }^{b}$ Laboratoire LIENSs Littoral Environnement et Sociétés, Institut écologie et environnement (IEE), French National Centre for Scientific Research (CNRS), Paris, France. ${ }^{c}$ UNC Eshelman School of Pharmacy, University of North Carolina (UNC), Chapel Hill , North Carolina 27599, USA. ${ }^{d}$ CNRS, Directrice de Recherche, Prof. Université Paris-Saclay, Paris, France. ${ }^{e}$ Dept. of Chemistry and Biochemistry, Interdisciplinary Center for Nanotoxicity (ICN), Jackson State University (JSU), USA. ${ }^{f}$ Dept. of Chemistry and Biochemistry, Faculty of Sciences, University of Porto, Porto, Portugal. ${ }^{g}$ Department of Pharmacology and Toxicology, University of Texas Medical Branch, Galveston, TX, USA. ${ }^{h}$ Department of Coatings and Polymeric Material, North Dakota State University (NDSU), Fargo, USA. ${ }^{i}$ BIOFISIKA: Basque Center for Biophysics (CSIC-UPV/EHU), Basque Country, Spain. ${ }^{j}$ Abteilung für Organische Chemie Universität Rostock, Rostock, Germany. ${ }^{k}$ European Bioinformatics Institute (EMBL-EBI), Cambridge, United Kingdom. ${ }^{l}$ Dept. of Mathematics, Miami Dade Colege (MDC), Miami, FL, USA. ${ }^{\mathrm{m}}$ Dept. of Systems and Comput. Eng., Carleton University, Canada. ${ }^{n}$ Dept. of Org. and Inorg. Chem., Univ. of Basque Country (UPV/EHU), Basque Country, Spain. ${ }^{\circ}$ United States Environmental Protection Agency (EPA), Ohio, USA. ${ }^{p}$ Faculty of Medicine and Health Technology, Tampere University, Tampere, Finland. ${ }^{q}$ Cheminformatics Lab., Universidade Federal da Paraiba (UFPB), Brazil. ${ }^{r}$ IKERBASQUE, Basque Foundation for Science, 48011, Bilbao, Biscay, Basque Country, Spain. 


\section{Welcome from chairs.}

Dear colleagues worldwide, we invite you to MOL2NET-07, Conference on Molecular, Biomedical, and Computational Sciences and Engineering (Formerly International Conference on Multidisciplinary Sciences), ISSN: 2624-5078, MDPI SciForum, Basel, Switzerland, 2021. MOL2NET is an international conference formed by an association of several inter-university tans-Atlantic congresses (Click

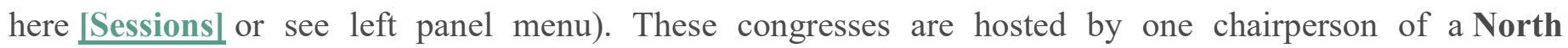
America/Europe university and one or various co-host chairpersons from other centers worldwide. Topics of interest. Even when the conference as a whole is multi-disciplinary each congress normally focus on specific niches of interest at interface of Chemistry, Molecular Engineering, Biomedical Engineering, or Computational Sciences and Engineering. As results the topics of interest are wide and range from the study of small molecules phenomena to the behavior of large complex networks in nature and society. This include but is not limited to, Chemistry (All areas), Mathematics (Applied), Physics (Applied), Materials Science, Nanotechnology, Biology and Life Sciences (All areas), Medicine, Biomedical Engineering, Education, along with Computer Sciences, Data Analysis, Statistics, Artificial Intelligence, Deep Learning, Bioinformatics, Systems Biology, and Complex Networks Sciences.

MOL2NET Congresses (Sessions). This a multidisciplinary conference formed by various interrelated congresses (Click here [Sessions] or see left panel menu). Consequently, most congresses share topics. From previous section you can conclude that the topics of interest covered are wide. However, you can find thematic congresses focused closely related areas of interest. You can participate both online or in person (face-to-face) in some of the congresses we organize in different universities worldwide (see sessions in the conference menu). Publication of all communications of the congresses will be online via the platform SciForum. We welcome proposals for organization of congresses in different universities. Please, do not hesitate to contact conference chairperson and/or scientific committee presidents. CHEMBIOMOL congress focus on Chemical Synthesis, $\underline{\text { Medicinal Chemistry, Vaccine }}$ $\underline{\text { Design, }}$ Biotechnology, and related areas. NANOBIOMAT congress focus on Nanotechnology, Materials $\underline{\text { Sciences, }}$ Polymers, Physical Chemistry, and related areas. CHEMBIOINFO congress focus on Cheminformatics, Bioinformatics, Drug Discovery, Quantum Chemistry, etc. AIMEDIC congress

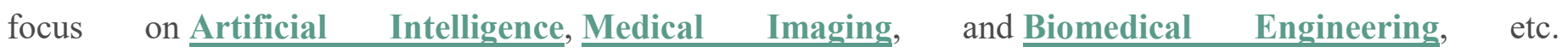

Conference schedule. The conference runs as year-round conference series with two main stages. These stages are: (1) Call for papers and (2) Post-publication reviews. In the first stage we open independent congresses in person and/or online, receive papers, carry out pre-publication review of papers, and perform online publication. Please, read the [MOL2NET Publication Schedule]. Consequently, submissions are open to be published asap upon acceptance in Sciforum platform almost all year at no cost for authors (year-round and free of cost publication). Depending on the schedule we also run a second stage called [MOL2NET Post-Publication Schedule]. On the Post-publication stage we close submission of papers and invite all members of committee, authors, and validated social network followers, to become post-publication reviewers. In this moment members of all workshops can interact together on brainstorming sessions making online comments each other. 
Headquarters and Supporters. MOL2NET runs both online and/or in person congresses in host

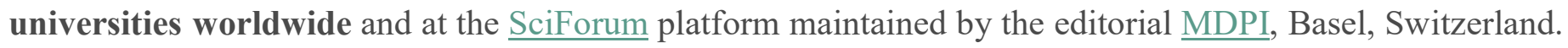
The idea of this multidisciplinary conference emerged from the melting pot formed as the result of multiple collaborations of professors from many centers worldwide.

International Partners. Internationally, professors and researchers world around are the main partners, cohost chairs, and/or committee members. From North America side's researchers from Stanford Center for Biomedical Informatics Research ( $\underline{\text { BMIR }}$ ), Stanford University (TANFORD), USA; Center for the Study of Biological Complexity ( $\underline{\text { CBDS }}$ ) of Virginia Commonwealth University ( $\underline{\text { VCU }})$, USA; Miami Dade College (MDC), USA; North Dakota State University (NDSU), USA; Jackson State University (JSU), USA, and Carleton University (CARLETON), Canada, among others, are hosts of our congresses. From Europes's side researchers and/or professors from European Bioinformatics Institute (EMBL-EBI) Cambridge, University of Birmingham (UBIRMINGHAM), University of Portsmouth (UPORT), United Kingdom, Universität Rostock Institut für Chemie (UROSTOCK), Germany, Centre National de la Recherche Scientifique (NRS), and Université Paris-Saclay ( $\underline{\mathbf{U P S}})$, Paris, France, are some of the co-hosts of our congresses. In addition, other institutions are co-founders and/or supporters of this conference worldwide, plz see full list on committee.

Headquarters \& Local Partners. The Local Headquarters (HQs) of this conference series are in the Faculty of Science and Technology, University of Basque Country ( $\underline{\text { UPV/EHU) }}$, Basque Country, Spain. More specifically, the founders and strongest supporters of the conference are professors endowed by IKERBASQUE, Basque Foundation for Sciences (IKERBASQUE) and professors from the Dept. of Org. \& Inorg. Chem. In addition, professors affiliated to Basque Centre for Biophysics (BIOFISIKA) of the University of Basque Country (UPV/EHU), and Center for Cooperative Research in Biosciences (CICBIOGUNE), with ties to previous departments are also supporters of this conference. Last, professors from the Department of Computer Sciences of the University of Coruña (UDC), Galicia, Spain act also as local co-hosting partners. Please, see full committees lists [MOL2NET HONOR \& SCI COMMITTEE] and [MOL2NET-IKERBASQUE Staff]. In this sense, all these and other local and international centers acts as co-hosting partners for some of the workshop sessions of this conference.

MOL2NET Lemma and Topics of Interest. The acronym MOL2NET summarizes the running title of this conference: FROM MOLECULES TO NETWORKS. This lemma is inspired by the possibility of spreading the knowledge about multiple topics by promoting multidisciplinary collaborations in science with conferences including topics ranging from quantum world to large social networks. In this sense, the spirit of this conference is in consonance with the lemma EMAN TA ZABAL ZAZU (Give and extend knowledge). This anthem from the 19th century is written in Basque language (Euskera) and is used now a days as the official motto of the University of Basque Country (UPV/EHU). We suggest you to download the template to write your communications for conference workshops Whoever, some other congresses of the series have their custom templates. Please, read carefully the [Instructions to authors] about publication model, copyright, authors responsibilities, etc. 
MOL2NET-MDPI JCR Journals Issues. In parallel, the members of committees and/or authors are encouraged to edit special issues for different journals of the editorial MDPI (http://www.mdpi.com/). The special issue is now in call for papers, submissions are welcome in a posteriori, in parallel, or totally independently from the conference. Manuscripts should be submitted online at www.mdpi.com by registering and logging in to this website.

In order to send a proposal of associated workshop and/or special issue contact email: mol2net.chair@gmail.com. 